\title{
A RETROSPECTIVE ANALYSIS ON PHARMACOLOGICAL APPROACHES TO COVID-19 PATIENTS IN AN ITALIAN HUB HOSPITAL DURING THE EARLY PHASE OF THE PANDEMIC
}

\author{
G. Menardi ${ }^{1}$, L. Infante ${ }^{2}$, V. Del Bono ${ }^{3}$, L. Fenoglio ${ }^{4}$, D. Collotta ${ }^{1}$, P. Macagno ${ }^{5}$, \\ C. Bedogni ${ }^{5}$, M. Rebora ${ }^{5}$, C. Fruttero ${ }^{2}$, M. Collino ${ }^{6}$ \\ ${ }^{1}$ Department of Drug Science and Technology, University of Turin, Turin, Italy \\ ${ }^{2}$ Hospital Pharmacy, S. Croce e Carle Hospital, Cuneo, Italy \\ ${ }^{3}$ Division of Infection Disease, $S$. Croce e Carle Hospital, Cuneo, Italy \\ ${ }^{4}$ Division of Internal Medicine, S. Croce e Carle Hospital, Cuneo, Italy \\ ${ }^{5}$ General Manager, S. Croce e Carle Hospital, Cuneo, Italy \\ ${ }^{6}$ Rita Levi-Montalcini Department of Neuroscience, University of Turin, Turin, Italy \\ E-mail: massimo.collino@unito.it
}

Doi: $10.36118 /$ pharmadvances.2021.15

\begin{abstract}
SUMMARY
COVID-19 is a complex, multi-organ systemic disease caused by the SARS-CoV-2 coronavirus whose pathophysiological basis and pharmacological approaches are still to be defined. Our study aims to retrospectively evaluate the role played by clinical and demographic variables as well as the specific therapies administered in COVID-19 patients admitted to a referral Hospital in Northern Italy (Santa Croce e Carle Hospital, Cuneo) during the COVID-19 first epidemic wave. We analyzed data from 277 patients with virological diagnosis of COVID-19 who were admitted to the hospital from February $20^{\text {th }}$ to April $30^{\text {th }}$ 2020. The inpatients' pharmacological treatments and clinical outcomes were assessed up to May $31^{\text {st }}$ 2020. Correlations between pharmacological treatments as well as clinical and demographic variables and clinical outcomes have been performed.

Several drugs were used in different dosages and combinations. In the first weeks of the epidemic, the most widely used drugs were hydroxychloroquine and the antivirals lopinavir/ritonavir and darunavir/cobicistat, then replaced by corticosteroids and heparin derivatives. Supplementation with vitamin C, D, or polyvitamins exerted a slight, but not significant, increase in survival rate, mainly in patients over 65 years old. Results showed that age and comorbidities significantly affected the survival as shown by the Charlson Comorbidity Index which was significantly higher in patients who died than in those who survived, thus confirming that victims of the disease were mostly debilitated and elderly people.

The most influencing COVID-19 survival factors here recorded were age and comorbidities. No statistically significant differences were detected among the different pharmacological treatments used in our clinical setting during the first epidemic wave.
\end{abstract}

\section{Key words}

COVID-19; Italian hub hospital; pharmacological treatments; comorbidities.

\section{Impact statement}

No significant differences have been recorded in terms of efficacy among the several pharmacological approaches used during the first epidemic way in an Italian hub hospital. 


\section{INTRODUCTION}

SARS-CoV-2 infection, which triggers the COVID-19 disease, originated in Wuhan, China at the end of 2019. Subsequently, it spread to Europe and the rest of the world. Italy was one of the first country affected by SARS-CoV-2 epidemic having the patient 1 been detected in Lombardy region on February $20^{\text {th }}$ 2020. The first wave of the COVID-19 epidemic in Italy lasted from February $20^{\text {th }}$ to the end of May and it was characterized by a very rapid spread of cases and deaths and a strong territorial concentration in the north of the country (1). Piedmont Region, in the North-western part of Italy, had registered 30637 infections and 3867 deaths, but there may had been more, according to a seroprevalence survey conducted by the Italian National Institute of Statistics (ISTAT) and the Italian Ministry of Health, suggesting that the ratio of notified to actual cases was at least 1 to 6. The pharmacological approach for treating SARS-CoV-2 infection has been proposed as a two-step approach. In the first step, antiviral drugs are used due to their alleged inhibitory effect on viral entry and replication, whereas in the second step, which typically begins after 7-10 days from the onset of symptoms, immunosuppressive and immunomodulatory drugs are thought to be of benefit because of the hyperinflammatory and cytokine release syndrome (2). However, the medications used in the first wave of the epidemic were based on a very limited experience, mainly carried out on other coronavirus-linked diseases, such as SARS and MERS. Here we report the results of a retrospective analysis, conducted on 277 inpatients with confirmed COVID-19 admitted to one of the largest referral hospital in Piedmont, the Santa Croce e Carle Hospital in Cuneo. The study describes the pharmacologic approaches recorded during the early phase of the epidemic as well as the most relevant factors affecting patients' survival in the selected population.

\section{MATERIALS AND METHODS}

We analysed data from patients with laboratory confirmed COVID-19 who were admitted to the Department of Medicine of Santa Croce e Carle Hospital (Cuneo, North West Italy) from February $20^{\text {th }} 2020$, the date of the first Coronavirus infection in Italy, to April 30 2020. The inpatients' pharmacological treatments and clinical outcomes were assessed up to May $31^{\text {st }} 2020$.

A descriptive analysis was performed to assess the distribution of inpatients demographic characteristics, clinical characteristics (comorbidities and complications), drugs and supplements administered during hospitalization and clinical outcomes in terms of survival status.

Data were analysed and presented as frequencies and percentages and compared for any significant difference using chi square or Fischer's exact test and Odds Ratios (ORs) with $95 \%$ confidence interval $(95 \% \mathrm{Cl})$. Continuous variables were analysed and presented as median and interquartile ranges and means with standard deviations where appropriate, compared for any significant difference using the t-test. P-values less than 0.05 were considered statistically significant. The Charlson Comorbidity Index $(\mathrm{CCl})$ has been calculated using an online calculator which included the age implementation (www.mdcalc.com/charlson-comorbidity-index-cci\#next-steps).

\section{RESULTS}

\section{Demographic characteristics}

277 patients have been included in the retrospective analysis. As reported in table I, the mean age was 69.2 years with a median of 70 years and an interquartile range (IQR) of 59-82 with a large majority of male patients (62.8\%). $81.6 \%$ of patients (137 men and 89 women) survived, whereas 14 females and 37 males died, with no gender-related significant differences. 
Table I. Characteristics of COVID-19 inpatients.

\begin{tabular}{|c|c|c|c|c|c|}
\hline \multicolumn{2}{|c|}{ Characteristics } & \multirow{2}{*}{$\begin{array}{c}\begin{array}{c}\text { Overall Sample } \\
\mathbf{2 7 7}\end{array} \\
69.2 \\
\pm 15.5 \\
\end{array}$} & \multirow{2}{*}{$\begin{array}{c}\text { Survived (\%) } \\
\text { 226 (81.6) } \\
67.2 \\
\pm 15.6\end{array}$} & \multirow{2}{*}{$\begin{array}{c}\text { Deceased (\%) } \\
\mathbf{5 1 ( 1 8 . 4 )} \\
77.9 \\
\pm 11.4 \\
\end{array}$} & \multirow{3}{*}{$\begin{array}{c}\begin{array}{c}P \\
\text { Value }\end{array} \\
<0.01\end{array}$} \\
\hline \multirow{7}{*}{ Age } & $\begin{array}{l}\text { Mean } \\
\pm \text { SD }\end{array}$ & & & & \\
\hline & Median (IQR) & $\begin{array}{c}70 \\
(59-82)\end{array}$ & $\begin{array}{c}67 \\
(57-79) \\
\end{array}$ & $\begin{array}{c}80 \\
(73-87)\end{array}$ & \\
\hline & $18-34(\%)$ & $7(2.5)$ & $7(3.1)$ & $0(0)$ & \multirow{5}{*}{$<0.01$} \\
\hline & $35-49(\%)$ & $25(9.0)$ & $23(10.2)$ & $2(3.9)$ & \\
\hline & $50-64(\%)$ & $73(26.4)$ & $68(30.1)$ & $5(9.8)$ & \\
\hline & 65-79 (\%) & $90(32.5)$ & $72(31.9)$ & $18(35.3)$ & \\
\hline & $\geq 80(\%)$ & $82(29.6)$ & $56(24.8)$ & $26(51.0)$ & \\
\hline \multirow{2}{*}{ Sex } & Men (\%) & $174(62.8)$ & $137(78.7)$ & $37(21.3)$ & \multirow{2}{*}{$>0.05$} \\
\hline & Women (\%) & $103(37.2)$ & $89(86.4)$ & $14(13.6)$ & \\
\hline
\end{tabular}

A single tailed Student Test has been applied for the comparison of age and sex between the surviving and the deceased patients. A chi-square test has been performed for the statistical analysis of the age groups dependency.

As shown in figure 1, patients survival was age-dependent: more than $90 \%$ of patients in the age range between 18 and 64 years survived, whereas the percentage of survivors was reduced to $74 \%$ in older patients $(O R=$ $4.81 ; \mathrm{Cl} 95 \%(2.08-11.15))$.

\section{Treatments}

During the early phase of COVID-19, different pharmacological therapies have been pro- posed. As shown in Figure 2, one of the first drug used in our setting was hydroxychloroquine, starting on March $5^{\text {th }}$ up to May $26^{\text {th }}$ 2020. Treatment consisted of high initial doses (400-800 mg/day), followed by $200 \mathrm{mg} /$ day maintenance dose. On May 29th, the Italian Medicine Agency ruled out the use of hydroxychloroquine and chloroquine in COVID-19 patients, except for inpatients under close monitoring involved in clinical trials or nation-

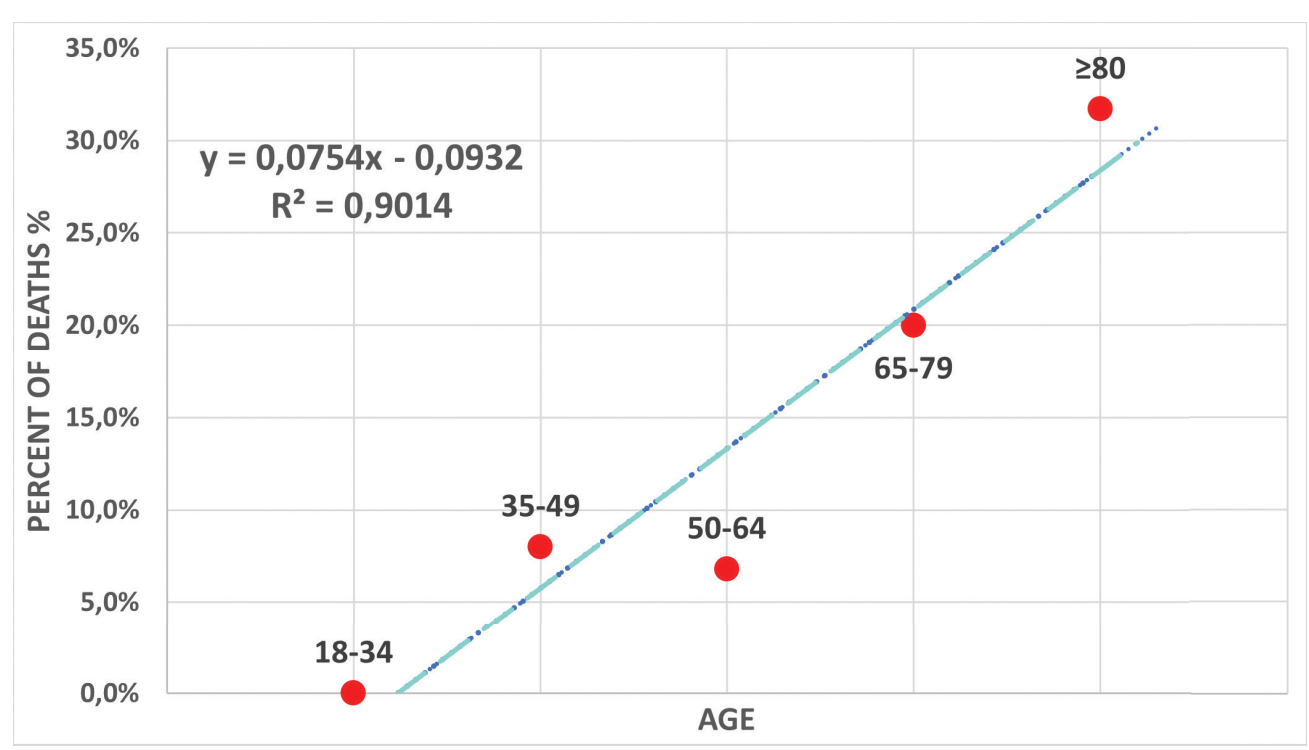

Figure 1. Regression line showing the relationship between age and the percent of deaths. 


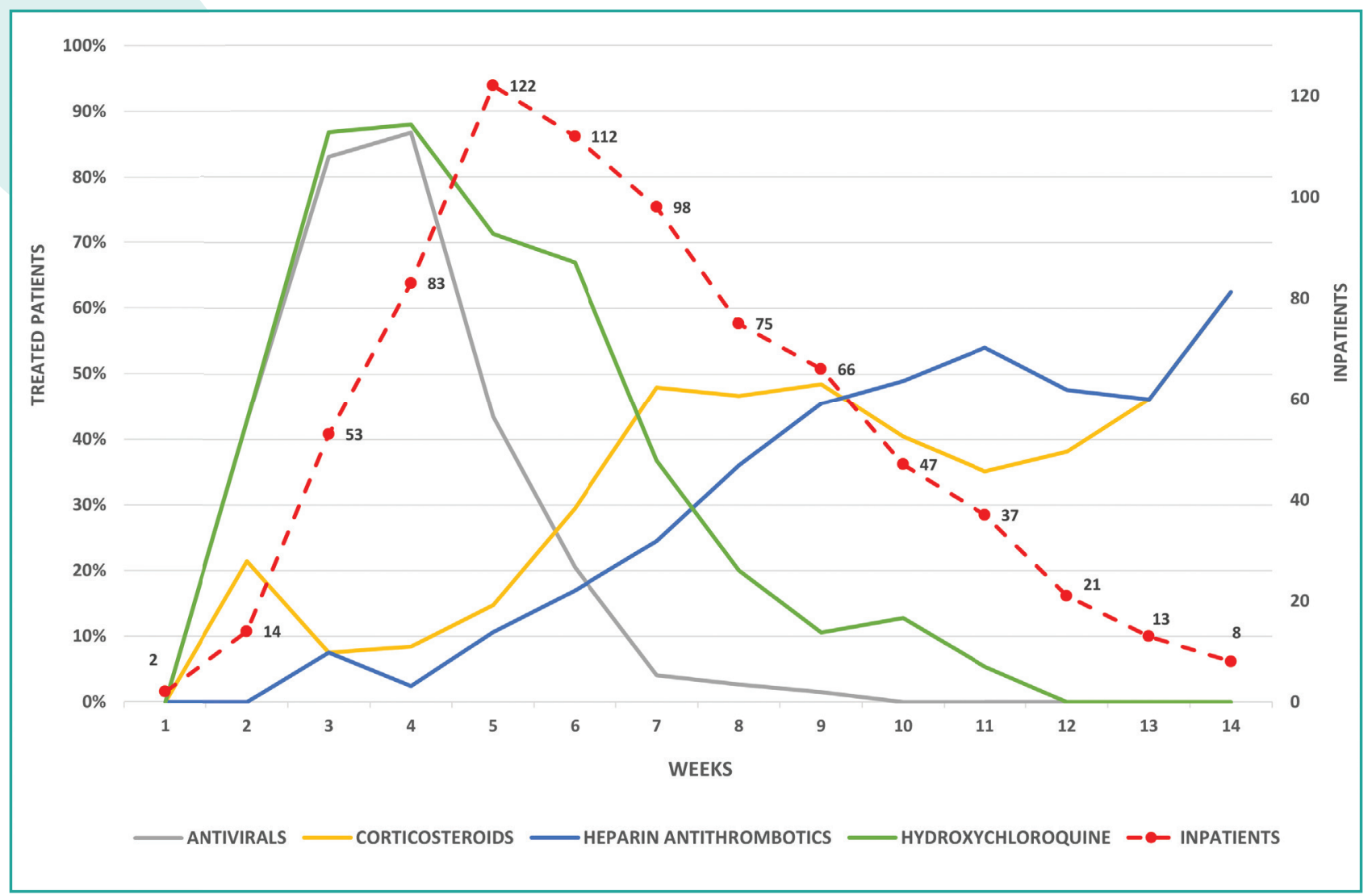

Figure 2. Pharmacological treatments administered to laboratory-confirmed COVID-19 patients hospitalized in the Medical Department.

al emergency management programmes. As shown in figure 2, the administration of the antivirals lopinavir/ritonavir and darunavir/cobicistat fell sharply in April 2020. This is due both to limited availability on the market and the limitations of their use in combination with other pharmacological therapies because of their potential high incidence of drug interactions. In addition, several studies published in the same period showed that they were not effective against COVID-19 (2). Subsequently, the use of corticosteroids, mainly dexamethasone, prednisone and methylprednisolone increased. The most widely used antibiotics were beta-lactams, either carbapenems (ertapenem, meropenem), or cephalosporins (mainly ceftriaxone), or piperacillin/tazobactam. Macrolides (azithromycin), and tetracyclines (doxycycline) were also administered to COVID-19 patients. More than $80 \%$ of COVID-19 patients were treated with antithrombotic heparin deriva- tives either at prophylactic dosages (61.7\%) or at therapeutic dosages (23.1\%), according to the severity of respiratory failure and the risk of pulmonary embolism. Prophylaxis consisted of a single daily administration of low molecular weight heparin $(\mathrm{LMWH})$, enoxaparin and parnaparin, at dosages up to 6,000 IU/day, unfractionated heparin (UFH) at dosages up to $12,500 \mathrm{IU} /$ day or fondaparinux at dosages up to $2.5 \mathrm{mg} /$ day. In total, 64 patients were treated with high doses of heparin derivatives, including enoxaparin, unfractionated heparin and fondaparinux, with a survival rate over $80 \%$.

As shown in table II, correlations between the patient survival and the drug treatments showed no significant differences among the proposed therapies, nor alone neither in combination. The association of hydroxychloroquine with antithrombotic heparin derivatives showed a greater efficacy - although not significant - compared to other treatment 
Table II. Pharmacological treatments administered to inpatients with confirmed COVID-19.

\begin{tabular}{|c|c|c|c|c|c|c|}
\hline \multicolumn{2}{|c|}{ Treatments } & $\begin{array}{l}\text { Overall } \\
\text { sample } \\
N=277\end{array}$ & $\begin{array}{c}\text { Survived } \\
N=226 \\
(81.6)\end{array}$ & $\begin{array}{c}\text { Deceased } \\
N=51 \\
(18.4)\end{array}$ & $\begin{array}{c}\% \\
\text { Patients } \\
\text { in ICU }\end{array}$ & $\begin{array}{c}P \\
\text { Value }\end{array}$ \\
\hline \multirow{4}{*}{$\begin{array}{l}\text { Single } \\
\text { Therapy }\end{array}$} & Corticosteroids & $85(30.7)$ & $63(74.1)$ & $22(25.9)$ & 16.5 & \multirow{4}{*}{$>0.05$} \\
\hline & Heparin antithrombotics & $64(23.1)$ & $52(81.3)$ & $12(18.7)$ & 17.2 & \\
\hline & Hydroxychloroquine & $200(72.2)$ & $168(84.0)$ & $32(16.0)$ & 16.5 & \\
\hline & Antivirals & $116(41.9)$ & $97(83.6)$ & $19(16.4)$ & 19.8 & \\
\hline \multirow{5}{*}{$\begin{array}{l}\text { Combined } \\
\text { Therapy }\end{array}$} & Hydroxychloroquine+antivirals & $112(40.4)$ & $93(83.0)$ & $19(17.0)$ & 19.6 & \multirow{5}{*}{$>0.05$} \\
\hline & Hydroxychloroquine+corticosteroids & $60(21.7)$ & $47(78.3)$ & $13(21.7)$ & 18.3 & \\
\hline & $\begin{array}{l}\text { Hydroxychloroquine+heparin } \\
\text { antitrombotics }\end{array}$ & $35(12.6)$ & $30(85.7)$ & $5(14.3)$ & 11.4 & \\
\hline & Antivirals+corticosteroids & $26(9.4)$ & $20(76.9)$ & $6(13.1)$ & 19.2 & \\
\hline & $\begin{array}{l}\text { Corticosteroids+heparin } \\
\text { antitrombotics }\end{array}$ & $25(9.0)$ & $18(72.0)$ & $7(28.0)$ & 12.0 & \\
\hline
\end{tabular}

Statistical analysis was performed by using the chi-square test.

schedules. The majority of patients were administered hydroxychloroquine in combination with corticosteroids or antivirals, and received an antithrombotic prophylaxis using a low dosage of LMWH, UFH or fondaparinux. The implementation of intermediate or therapeutic doses in 64 patients did not reduce the odds of develop thromboembolic and cardiac complications (OR = 0.90; Cl 95\% (0.43-1.86)). Even when patients were stratified according their age, no significant differences in efficacy among treatments were recorded. Nevertheless, age remains the most relevant factor affecting the clinical outcome and in-hospital death. Inpatients treated with antiviral and hydroxychloroquine had the lowest mean age than the group exposed to other COVID-19 therapies $(p<0.01)$ and were also characterised by a shorter hospitalisation time ( $p<$ $0.05)$. Therapy was supplemented with vitamin C, D, polyvitamins in 68 patients and the best outcome was recorded in patients over 65 (84\% survival), when compared to patients of the same age but not treated with vitamins (70\% survival, Fisher's exact test $p=0.0565$ ).

\section{Clinical characteristics}

The impact of comorbidities on patient survival was also analysed (table III). More than
$30 \%$ of patients was suffering of at least one comorbidity and there was a robust increase in the death risk in the presence of comorbidities (OR $=5.93 ; 95 \% \mathrm{Cl}(3.08-11.42))$. We identified 17 patients suffering from chronic cardiovascular diseases, 17 patients affected by chronic respiratory diseases, resulting in an in-hospital death of 7 patients per group $(O R=3.44 ; 95 \% \mathrm{Cl}$ (1.24-9.52)). There were 22 diabetic patients, 1 with type 1 diabetes and 21 with type 2 diabetes, with an in-hospital death of 7 patients in the latter group $(\mathrm{OR}=2.73 ; 95 \% \mathrm{Cl}(1.03-7.24))$. Hypoglycaemic drugs (insulin and/or oral hypoglycaemic drugs) have been also administered to further 29 patients due to transient hyperglycaemic state, 9 of which died (OR $=2.46 ; 95 \%$ $\mathrm{Cl}$ (1.03-35.83)). Patients taking hypoglycaemic drugs were older than those who did not received the hypoglycaemic treatments. The $\mathrm{CCl}$ was significantly higher for the deceased than for the survivors.

Patients with cancer had higher increase of death than other populations (OR $=5.17 ; 95 \%$ $\mathrm{Cl}$ (1.94-13.78)).

As shown in figure 3 , the numbers of comorbidities and complications occurred during the hospitalization significantly affected patients' survival. 
G. Menardi, L. Infante, V. Del Bono, et al.

Table III. Comorbidities in COVID-19 inpatients.

\begin{tabular}{|c|c|c|c|c|c|}
\hline \multicolumn{2}{|c|}{ Clinical characteristics } & \multirow{2}{*}{$\begin{array}{c}\begin{array}{c}\text { Overall } \\
\text { sample } \\
\mathbf{N}=277\end{array} \\
201(72.6)\end{array}$} & \multirow{2}{*}{$\begin{array}{c}\begin{array}{c}\text { Survived } \\
\mathbf{N}=\mathbf{2 2 6 ( 8 1 . 6 )}\end{array} \\
166(82.6)\end{array}$} & \multirow{2}{*}{$\begin{array}{c}\text { Deceased } \\
\mathbf{N}=\mathbf{5 1 ( 1 8 . 4 )} \\
35(17.4)\end{array}$} & \multirow{3}{*}{ P Value } \\
\hline \multirow{4}{*}{$\begin{array}{l}\text { Admitting } \\
\text { diagnosis }\end{array}$} & Pneumonia (\%) & & & & \\
\hline & Virus disease (\%) & $24(8.7)$ & $24(100)$ & $0(0.0)$ & \\
\hline & Lung failure (\%) & $14(5.1)$ & $6(42.9)$ & $8(57.1)$ & \multirow{2}{*}{$<0.01$} \\
\hline & Other (\%) & $38(13.7)$ & $30(78.9)$ & $8(21.1)$ & \\
\hline \multirow{5}{*}{ Comorbidities } & $\begin{array}{l}\text { Chronic cardiovascular } \\
\text { diseases (OR) }\end{array}$ & 17 & 10 & $7(3.44)$ & $<0.05$ \\
\hline & $\begin{array}{l}\text { Chronic respiratory } \\
\text { diseases }(O R)\end{array}$ & 17 & 10 & $7(3.44)$ & $<0.05$ \\
\hline & Type 2 diabetes (OR) & 21 & 14 & $7(2.73)$ & $<0.05$ \\
\hline & Cancer (OR) & 18 & 9 & $9(5.17)$ & $<0.05$ \\
\hline & $\begin{array}{l}\text { Charlson Comorbidity } \\
\text { Index Mean } \pm \text { SD }\end{array}$ & $3.1 \pm 2.1$ & $2.8 \pm 2.1$ & $4.7 \pm 1.9$ & $<0.01$ \\
\hline \multirow{6}{*}{$\begin{array}{l}\text { Acute } \\
\text { complications }\end{array}$} & Respiratory failure & $157(56.7)$ & $121(77.1)$ & $36(22.9)$ & \multirow{6}{*}{$>0.05$} \\
\hline & Acute CVD & $38(13.7)$ & $25(65.8)$ & $13(34.2)$ & \\
\hline & Sepsis and septicemic & $20(7.2)$ & $10(50.0)$ & $10(50.0)$ & \\
\hline & Pulmonary embolism & $12(4.3)$ & $10(83.3)$ & $2(16.7)$ & \\
\hline & Kidney failure & $8(2.9)$ & $8(100.0)$ & $0(0.0)$ & \\
\hline & $\begin{array}{l}\text { Acute cerebrovascular } \\
\text { diseases }\end{array}$ & $8(2.9)$ & $2(25.0)$ & $6(75.0)$ & \\
\hline
\end{tabular}

The chi-square test was applied for the statistical analysis of the "admitting diagnosis", "comorbidities" and "acute complications". The impact on patient survival has been calculated using OR and its IC has been expressed by multiplying its Standard Error for 1.96 assuming a normal distribution.

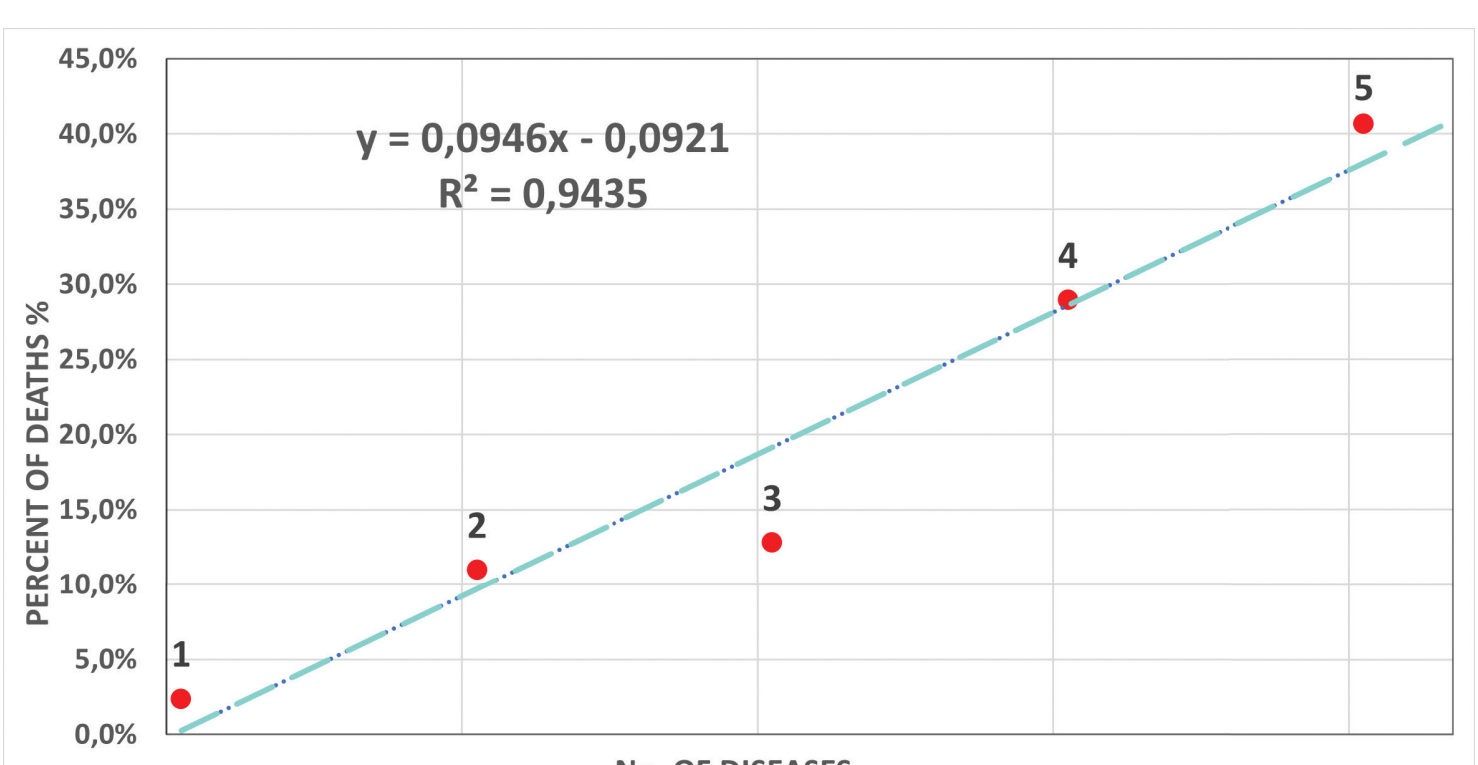

No. OF DISEASES

Figure 3. Regression line showing the relationship between number of diseases and the percent of deaths. 


\section{DISCUSSION}

This retrospective analysis, carried out on 277 laboratory-confirmed COVID-19 patients hospitalized in the Medical Department confirmed and further extended previous findings, showing that age and number of concurrent diseases are among the most important factors affecting survival from SARS-CoV-2 infection. Interestingly, despite our study was focused only on patients hospitalized in a Medical Department, the results we collected are in keeping with those carried out in the first wave of COVID-19 in Italy and other countries on ICU patients and which often included patients without laboratory-confirmed COVID-19 (3-6).

As shown in our study, during the early phase of COVID-19 epidemic, most of the inpatients were elderly individuals with severe COVID-19 and comorbidities. The progressive accumulation of senescent cells during life may play a role in the vulnerability of old people to COVID-19, resulting in reduced functionality of the organs, such as the lungs, and facilitating conditions for the development of fibrosis. Moreover, senescent cells can generate a pro-inflammatory environment, which might influence the expression of $\mathrm{CoV}$-associated molecules in human tissues, thus favouring viral entry $(3,4)$. Gender differences were also detected, with the highest prevalence of both admitted and deceased patients within the male population. The majority of the admitted patients were male in the age range 3580 , without any gender differences between the youngest and oldest groups. Our data on impact of gender as risk factor for COVID-19 morbidity and mortality are similar to those previously published (5-7).

Another important factor affecting the survival rate was the presence of concurrent diseases. The $\mathrm{CCl}$ mean was statistically higher in the deceased group than in the survived, showing that most victims of the virus were debilitated and elderly people. The highest frequency of in-hospital death was recorded in patients with $\geq 5$ concomitant diseases. The most relevant concomitant diseases were diabetes, chron- ic cardiovascular or respiratory diseases and cancer, being type 2 diabetes the one with the highest mortality risk. Similar results have been obtained in the French Coronado study and other retrospective analysis (8-11). The pathogenic mechanisms underlying the increased mortality in diabetic patients have not yet been elucidated, despite intriguing data suggest a pivotal role for the ACE2 receptor expressed by pancreatic islet cells, altering immune response and evoking activation of specific inflammatory pathways, thus, causing cytokine dysregulation $(9,12,13)$. Interestingly, a higher risk of mortality was also recorded in 29 non-diabetic patients who were given hypoglycaemic drugs (including insulin) due to transient hyperglycaemic conditions, most likely depending on the use of corticosteroid drugs. However, it must to be noted that the population under hypoglycaemic drugs was the oldest one and thus the increased mortality here recorded could be affected by age. The lack of data on Body Mass Index (BMI) and other anthropometric parameters does not allow further considerations on this specific issue.

Overall, the patients' survival was not affected by the specific drugs regiments, ranging from $72 \%$ for corticosteroids and heparin derivatives to $85 \%$ for hydroxychloroquine and heparin derivatives. The greater survival rate observed for patients taking hydroxychloroquine could conceivably explained by a sort of inclusion bias. In fact, that treatment was usually administered to patients with greatest odds of survival, such as those of younger age and with fewer comorbidities or those with less severe COVID 19. Under this light the age difference might explain by itself these differences, being patients given antivirals and hydroxychloroquine younger and with lower $\mathrm{CCl}$ than patients taking other COVID-19 therapies. Antithrombotic heparin drugs were administered to more than $80 \%$ of the patients either as prophylactic (61.7\%) and therapeutic (23.1\%) approaches. In order to contrast thromboembolic complications, the prophylactic dosages were increased, but the administration of in- 
termediate/ therapeutic doses did not reduce the odds of thromboembolic and cardiac complications. Additionally, no dose-dependent effects on mortality were recorded. Again, our data are in keeping with previously published and largest randomized, open-label trials (14-16). The impact of vitamin D deficiency on risk of COVID-19 severity and mortality as well as the effects of vitamin supplementation in COVID-19 patients has been already documented $(17,18)$. Here we observed a slight, despite no significant, improvement in patients' survival rate, mainly in over 65 , in the presence of supplementing therapies with vitamin $C, D$, or polyvitamins.

We are aware that the results of the present study must be interpreted with caution and a number of limitations should be borne in mind, including the limited number of patients, the lack of specific patients charactheristics such as the BMI, smoking habits and ethnicity. In

\section{REFERENCES}

1. Balasco N, D'Alessandro $V$, Ferrara $P$, Giovanni Smaldone, Luigi Vitagliano. Analysis of the time evolution of COVID-19 lethality during the first epidemic wave in Italy. Acta Biomed 2021;92(2):e2021171.

2. Sanders JM, Monogue LM, Jodlowski TZ, Cutrell JB. Pharmacologic Treatments for Coronavirus Disease 2019 (COVID-19): A Review. Jama 2020;323(18):1824-36.

3. Nicoli F, Solis-Soto MT, Paudel D, et al. Age-related decline of de novo $T$ cell responsiveness as a cause of COVID-19 severity. Geroscience 2020;42(4):1015-19.

4. Schultze, JL, Aschenbrenner AC. Cell, 2021;184(7):1671-92.

5. Su YJ, Kuo KC, Wang TW, Chang CW. Gender based differences in COVID-19. New Microbes New Infect 2021;100905.

6. Scully EP, Haverfield J, Ursin RL, Tannenbaum C, Klein SL. Considering how biological sex impacts immune responses and COVID-19 outcomes. Nat Rev Immunol, 2020;20(7):442-7. addition, we had no access to laboratory data such as blood glucose, creatinine clearance, coagulation indices, etc.

In conclusion, our study confirmed and further extended previous findings on the impact of age and concurrent diseases on COVID-19 morbidity and mortality, focusing on a well-selected population of laboratory-confirmed COVID-19 patients hospitalized in a Medical Department. Most notably, it offers a clear picture of the pharmacological treatments proposed during the first wave of the COVID-19 epidemic showing that changes in the drug treatments that took place in the early phase of the infection did not significantly affect patients's survival rate.

\section{CONFLICT OF INTERESTS}

The authors declare that they have no conflict of interests.

7. Mohamed, MO, Gale CP, Kontopantelis $\mathrm{E}$, et al. Sex Differences in Mortality Rates and Underlying Conditions for COVID-19 Deaths in England and Wales. Mayo Clin Proc 2020;95(10):2110-24.

8. Cariou, B, Hadjadj S, Wargny $M$, et al. Phenotypic characteristics and prognosis of inpatients with COVID-19 and diabetes: the CORONADO study. Diabetologia 2020;63(8):1500-15.

9. Boddu, SK, Aurangabadkar G, Kuchay MS. New onset diabetes, type 1 diabetes and COVID-19. Diabetes Metab Syndr 2020;14(6):2211-7.

10. Holman N, Knighton P, Kar P, et al. Risk factors for COVID-19-related mortality in people with type 1 and type 2 diabetes in England: a population-based cohort study. Lancet Diabetes Endocrinol 2020;8(10):823-33.

11. Williamson EJ, Walker AJ, Bhaskaran K, et al. Factors associated with COVID-19-related death using OpenSAFELY. Nature 2020;584(7821):430-6. 
12. Magliano DJ, Harding JL, Cohen $\mathrm{K}$, et al. Excess Risk of Dying From Infectious Causes in Those With Type 1 and Type 2 Diabetes. Diabetes Care 2015;38(7):1274-80.

13. Bertocchi I, Foglietta F, Collotta D, et al. The hidden role of NLRP3 inflammasome in obesity-related COVID-19 exacerbations: Lessons for drug repurposing. $\mathrm{Br} \mathrm{J}$ Pharmacol 2020;177(21): 4921-30.

14. Singh $B$, Ryan $H$, Kredo $T$, Chaplin $M$, Fletcher T. Chloroquine or hydroxychloroquine for prevention and treatment of COVID-19. Cochrane Database Syst Rev 2021;2(2):Cd013587.

15. Horby P, RECOVERY Collaborative Group, Lim ES, et al. Dexamethasone in Hospital- ized Patients with Covid-19. N Engl J Med 2021;384(8):693-704.

16. Bolzetta F, Maselli M, Formilan $M$, et al. Prophylactic or therapeutic doses of heparins for COVID-19 infection? A retrospective study. Aging Clin Exp Res 2021;33(1):213-7.

17. Getachew B, Tizabi Y. Vitamin D and COVID-19: Role of ACE2, age, gender, and ethnicity. J Med Virol 2021.

18. Annweiler G, Corvaisier M, Gautier J, et al. Vitamin D Supplementation Associated to Better Survival in Hospitalized Frail Elderly COVID-19 Patients: The GERIACOVID Quasi-Experimental Study. Nutrients 2020;12(11). 